\title{
Repurposing of Anti-diabetic Drug in Cancer Prevention
}

ISSN: 2637-773X

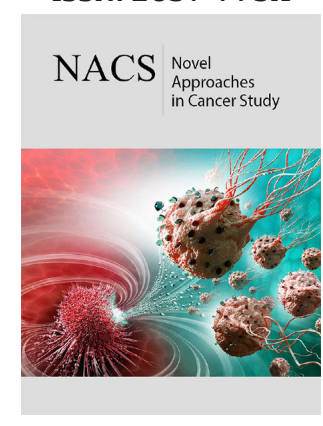

*Corresponding author: Ashish Bhattacharjee, Department of Biotechnology, National Institute of Technology, Durgapur-713209, West Bengal, India

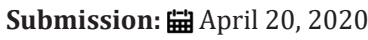

Published: 侮June 04, 2020

Volume 4 - Issue 5

How to cite this article: Chandreyee Datta, Payel Das, Ashish Bhattacharjee. Repurposing of Anti-diabetic Drug in Cancer Prevention. Nov Appro in Can Study. 4(5). NACS.000598. 2020. DOI: $10.31031 /$ NACS.2020.04.000598

Copyright@ Ashish Bhattacharjee, This article is distributed under the terms of the Creative Commons Attribution 4.0 International License, which permits unrestricted use and redistribution provided that the original author and source are credited.

\author{
Chandreyee Datta\# Payel Das \# and Ashish Bhattacharjee*
}

Department of Biotechnology, National Institute of Technology, Durgapur-713209, West Bengal, India

\# These authors contributed equally to this manuscript.

\begin{abstract}
Metformin is basically a biguanide derivative widely prescribed worldwide in the treatment of type II diabetes (T2D) and Polycystic Ovarian Diseases. Diabetes is a very common disease and can increase the chance occurrence of various types of cancers such as colon, rectum, pancreas and liver cancer compared to non-diabetic patients. Recently, epidemiological studies and meta analysis have demonstrated that T2D patients have comparatively lower incidence of tumor development than healthy individuals. Moreover, the patients diagnosed with cancer have lower mortality rate when treated with metformin, indicating a likely association between metformin and tumorigenesis. So far, metformin has been used in cancer prevention with reduced risk as an adjuvant or neoadjuvent drug. There are evidences from studies in diabetic cohort and laboratory studies that the action of metformin depends on a balance between concentration and exposure, which also depends crucially on cell and tissue specific pharmacological factors. In-vivo and in-vitro studies have revealed that metformin has a direct antitumor effect-which can depress tumor proliferation and induce apoptosis, autophagy and cell cycle arrest. Yet, there are several missing links regarding the role of drug transporters and drug-drug interactions, as well as the expression levels of transporters in normal versus tumor tissues which may affect patient exposure and dosing when metformin is used in cancer prevention. In this review, we mainly highlight different mechanistic pathways of anti cancerous outcome and new molecular target of metformin in cancer.
\end{abstract}

Keywords: Metformin; Phenformin; Biguanides; Breast cancer; Prostrate cancer; Lung (NSCLC) cancer, Diabetes

Abbreviations: T2D: Type II Diabetes; AMPK: Adenosine Monophosphate Activated Kinase; ATM: Ataxia Telengiectasis Mutated; LKB1: Liver Kinase B1; OS: Overall Survival; PFS: Progression Free Survival; NSCLC: Non-Small Cell Lung Cancer Cells

\section{Introduction}

Cancer is a disease in which abnormal and uncontrollable cell proliferation happens and it may involve adjacent tissue in the body. According to the 2018 reports, cancer is the second leading cause of death following heart disease globally [1]. According to the world scenario, WHO has reported that approximately one out of six people die from cancer around the world. Diabetes is a common disease of human life and can be an important factor for occurrence of various types of cancer such as colon, rectum, pancreas, and liver cancer compared to nondiabetic patients [2]. The most probable cause of higher occurrence of cancer in T2D patients is due to insulin resistance and mitogenic effect caused by hyperglycemia [2].

Biguanides are mainly of three types: Metformin, Phenformin, and Buformin. These biguanides were used for shorter period of time but since phenformin and buformin cause production of lactic acidosis they are not used anymore [3,4]. Metformin is a commonly used drug for treating diabetes now a days. Metformin was first introduced for therapeutic purpose in Dublin by Emile Werner and James Bell in the year 1922 [5]. They observed that this compound was capable of reducing blood glucose level in rabbits without affecting blood pressure and heart rate unlike other similar compounds [6]. Metformin acts to reduce glycogenesis through $5^{\prime}$ adenosine monophosphate activated kinase (AMPK) signalling and 
increases glucose uptake in muscle cells of diabetic patients which leads to a decrease in glucose and insulin levels [7-13]. In general, metformin inhibits mammalian target of rapamycin (mTOR) activity by activating ataxia telengiectasis mutated (ATM), Liver Kinase B1 (LKB1), and then AMPK -finally prevents protein synthesis and cell growth.

On chemical point of view, metformin is a synthetic biguanide and due to the presence of the polar guanidine fraction, biguanides are hydrophilic bases and exists as cationic species at physiological $\mathrm{pH}$ with a minimal expected passive membrane diffusion [14] Moreover, due to its positive charge, metformin accumulates within the matrix of mitochondria, and it inhibits complex I of the mitochondrial electron transport chain (ETC) resulting in a reduction in NADH oxidation and finally a reduction in the synthesis of ATP [15]. Researchers have also revealed that metformin binds poorly to the mitochondrial membrane compared to phenformin which may be one of the most important factor involved in the reduced risk of lactic acidosis compared to phenformin [16].

In case of T2D patients, who consume metformin orally, the concentration of metformin in the hepatic circulation may reach $50 \mu \mathrm{M}$; with the maximum plasma concentration of metformin at $20 \mu \mathrm{M}$ [17-19]. The hydrophilic and cationic nature of metformin at physiological pH makes it highly difficult for rapid diffusion through the cell membrane and exerts its effect on cell function. Moreover, the kidneys play a vital role in the elimination of unaltered metformin through the urine [20]. So, it is evident that for exerting it's biological function, metformin requires the presence and support of transporter molecules for its absorption, distribution, and elimination. In this regard, the organic cation transporters 1, 2, and 3 (OCT1, OCT2, and OCT3), the plasma membrane monoamine transporter (PMAT), and multidrug and toxin extrusion protein 1 and 2 (MATE1 and MATE2) transporters are reported to play key roles in transporting metformin into and out of the cell in the intestine, liver, and kidney [21-29]. The thiamine transporter 2 (THTR2) also plays a vital role in intestinal absorption and renal reabsorption of metformin [29]. Alterations in the OCT1 gene reduced hepatic uptake of metformin and reduced the efficacy of metformin in reducing blood glucose levels by inhibiting gluconeogenesis and glycogenolysis [30,31]. In this review article, we focused on the role of metformin in cancer prevention by highlighting its molecular mechanism of action and its effectiveness during clinical trials in cancer.

\section{Mechanism of action of metformin in cancer cells}

There are two general mechanisms like 'direct' and 'indirect' effect that could explain the putative anti-cancer effects of metformin. The 'indirect' anti-cancer effects of metformin arise from its ability to reduce insulin resistance, insulin levels, and fasting glucose levels [32]. Physiologically, insulin and insulin-like growth factor-1 (IGF1) largely regulate carbohydrate and lipid metabolism and storage and protein synthesis via transmembrane receptor binding and activation of receptor tyrosine kinase and subsequent activation of intracellular insulin receptor substrate-1 (IRS1). On the other hand, insulin and IGF1-mediated signalling pathways are also involved in pathogenesis and progression of several cancers via the activation of the Ras/Raf/MEK/ERK, PI3K/ Akt/mTORC1, and GSK3 $\beta / \beta$-catenin pathways $[17,33]$. The antiproliferative activity of metformin in several cancers is partly due to its ability to reduce the levels of insulin/IGF1, which in turn indirectly inhibits the insulin/IGF1 mediated molecular pathways that support tumor initiation and progression [33]. Metformin also exhibited 'direct' anti-cancer effects in many different cancer cells. Generally, cancer cells are known to utilize glucose rapidly through glycolysis (Warburg effect). To meet the energy needs of cancer cells compared to normal cells, metformin-mediated decrease in glucose levels should also curb tumor growth, although reports suggest that cancer cells use alternative sources of energy when starved of glucose or when glycolysis is inhibited [34-37].

In many cancer cell line, metformin-mediated activation of AMPK and subsequent modulation and regulation of intracellular proteins and their functions as anti-cancer/ anti-proliferative effect was observed [33,34,38-41]. Activation of AMPK in cancer cells is associated with inhibition of the mammalian target of rapamycin target 1 (mTORC1), c-Myc, and nuclear factor kappa light chain enhancer of activated B cells (NF- $\mathrm{B}$ ) pathways and activation of dsRNA ribonucleotide (DICER) and the tumor protein p53 (p53) pathway. All these pathways are known for exerting tumor suppressive, anti-proliferative, anti-migratory, and proapoptotic effects through various intracellular mediators, such as activation of anti-oncogenic genes, and downregulation of prooncogenic genes [42-50]. AMPK also phosphorylates and inhibits acetyl CoA carboxylase (ACC), thereby reducing lipid biosynthesis [51]. Inhibition of these anabolic processes of protein and lipid biosynthesis thus retards cancer cell growth and proliferation [52].

Metformin treatment is also associated with 'AMPK independent' anti-cancer effects. This is generally mediated by regulated in DNA Damage-1 (REDD1; also known as DNA damage inducible transcript-4-DDIT4), Rag GTPases, and signal transducer and activator of transcription-3 (STAT3). REDD1/DDIT4 is a known inhibitor of mTOR signalling and thereby possesses tumor suppressive properties by inhibition of protein synthesis and cell survival [53-56]. Researchers have also reported that metformin activated the p53/REDD1 axis to cause AMPK independent inhibition of mTOR in cancer cells [57]. Activation of the p53/ REDD1 pathway also reduces the expression of Cyclin D1, followed by reduction in cell proliferation [58]. These signalling events are summarized in Figure 1. On the other hand, metformin treatment inhibits the mTOR pathway via the inhibition of Rag GTPases in cancer cells, which in turn reduces protein synthesis and causes cell cycle arrest [58-60]. The abnormal activity of STAT3 has been reported in different cancer cells promoting the pro-oncogenic functions such as initiation, progression, and metastasis [61,62]. Overexpression of STAT3 contributes to cell survival, proliferation, cell cycle progression, anti-apoptosis, migration, invasion, angiogenesis, chemoresistance, immunosuppression, self-renewal and differentiation of stem cells by regulating the expression of its downstream target genes [61,62]. 


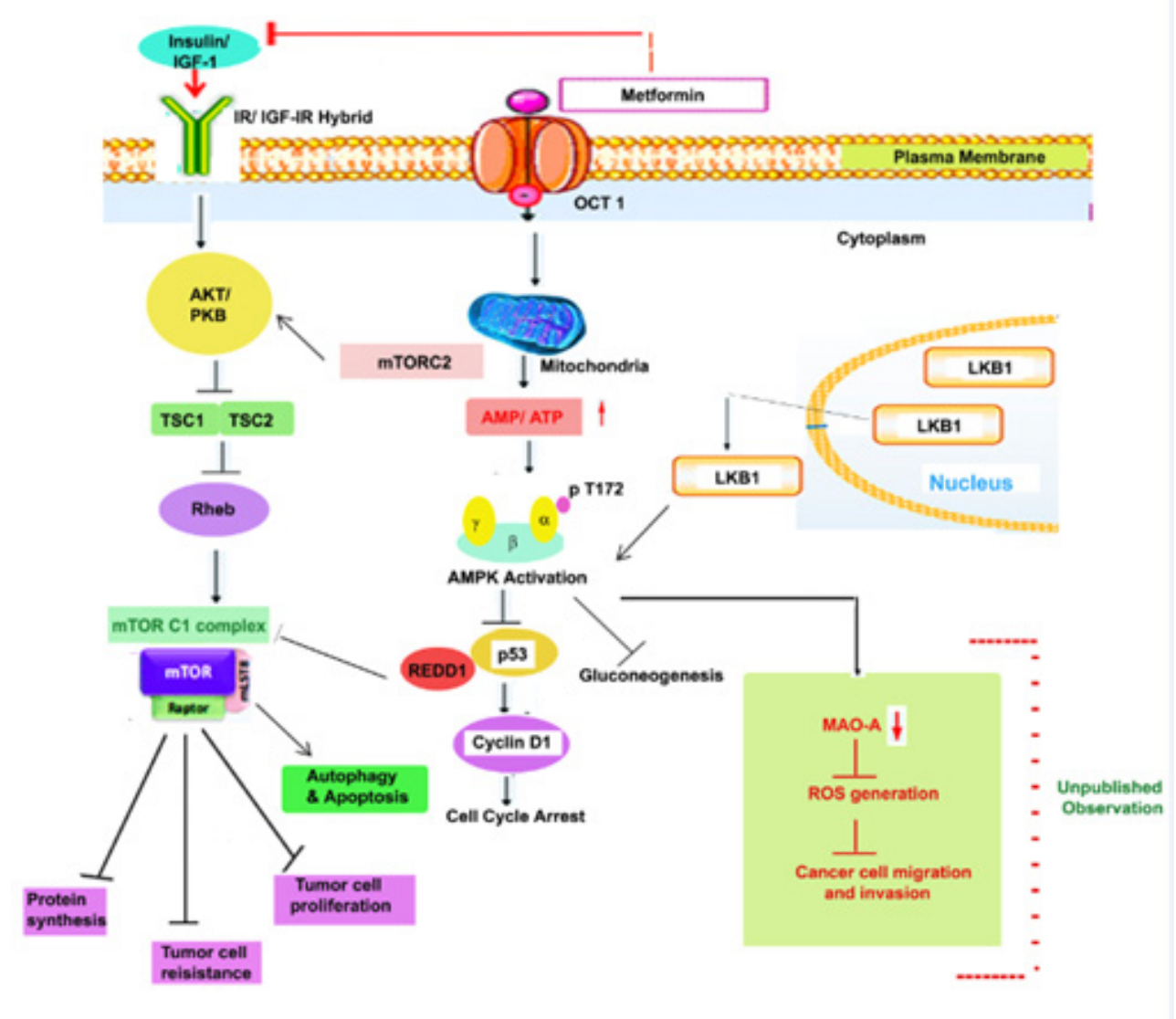

Figure 1: Molecular Mechanism of action of metformin in cancer cells.

General molecular mechanisms of metformin on inhibiting cancer cell growth. Metformin inhibits mitochondria complex 1 and activates adenosine monophosphate activated protein kinase (AMPK) signaling pathway, and/or inhibits the insulin signalling pathway. Metformin increases levels of AMP and thus promotes activation of AMPK through LKB1 phosphorylation of T172, which by controlling expression of p53 and cyclin D1 contributes in cell cycle arrest. Activation of AKT/PKB inhibits TSC1/TSC2 expression followed by activation of mTORC1 complex which helps in inhibition of protein cell synthesis, tumor cell resistance and tumor cell proliferation and activation of autophagic and apoptosis response. The green marked portion in this figure is indicating our unpublished observation, where we have seen that metformin mediated activation of AMPK can down regulate expression of MAO-A and this in turn contributes in down regulation of ROS and finally in the suppression of cancer cell migration and invasion.

IGF-1, insulin-like growth factor-1; IGF-1R, insulin-like growth factor-1 receptor; IR, insulin receptor; OCT1, organic cation transporter 1, TSC1 \& TSC2, Tuberous sclerosis $1 \& 2$; LKB1, Liver kinase B1; PKB, Protein kinase B.

The multifaceted ability of metformin to influence cancer cell growth and cancer progression through various molecular mechanisms, as discussed above, has made it an interesting candidate drug with potential in the treatment of cancer. In the following sections of the article, we briefly discuss the preclinical, and clinical studies that are currently testing metformin as a monotherapy or in combination with other chemotherapeutic drugs or phytochemicals/natural compounds for its efficacy as an anti-cancer/anti-tumor agent in the treatment of cancer. We will also discuss our unpublished observation in case of repurposing metfomin as anticancer drug in lung cancer treatment and probable new target of metformin in cancer treatment.

\section{Clinical trials using metformin}

Presently, there are several ongoing clinical trials in various stages that are evaluating metformin as a monotherapy or in combination with cytotoxic chemotherapy and/or radiotherapy for the treatment of cancer. The primary aim of these trials is to focus on establishing the effects of metformin on markers of cellular proliferation, pathological response rate, progression free survival, and recurrence free survival.

A sufficient amount of focus has been paid on investigating metformin as a potential anti-cancer agent for cases of breast cancer. Of these, two trials are carrying out using metformin as monotherapy. There are 9 more trials using metformin in combination with other anti-cancer agents such as capecitabine, cyclophosphamide, docetaxel, doxorubicin, erlotinib, epirubicin, exemestane, ganitumab, letrozole, sirolimus and temsirolimus. One trial is exploring the use of metformin plus atorvastatin combination as a possible treatment for breast cancer. In addition to survival outcomes, several well known markers of cancer like 
Ki67, S6 Kinase (S6K), Eukaryotic translation initiation factor binding protein 1 (4E-BP-1), AMPK are also being employed to study the effect of metformin on breast cancer. A phase 2 single arm window of opportunity trial of 39 breast cancer cases [63] showed significant reduction in Ki67 (36.5 to $33.5 \%$, p = 0.016) and an increase in terminal deoxynucleotidyl transferase dUTP nick-end labelling (TUNEL) staining (0.56 to $1.05, \mathrm{p}=0.004$ ) along with a significant fall in homeostatic model assessment (HOMA), used for determining the status of insulin resistance [64]. A recently published randomized control trial (RCT) also reported a decrease in Ki67 staining (mean $=3.4 \%, p=0.027$ ). Additionally, it noted an increase in mean AMPK score, fall in pAKT score and reduced caspase- 3 staining in patient samples with the use of metformin when compared to placebo [65]. A phase II RCT with 200 participants recorded no significant changes in Ki67 level on comparing metformin and placebo groups. But, interestingly, the cases with HOMA $\leq 2.8$ showed a non significant increase of Ki67 by $11.1 \%$ ( $95 \%$ Confidence interval (CI): $-0.6 \%$ to $24.2 \%$ ) and those with HOMA > 2.8 (implying a higher probability of insulin resistance) showed a non-significant mean proportional decrease in Ki67 by 10.5\% (95\% CI: $-26.1 \%$ to $8.4 \%$ ) [66]. Another non-randomized phase II trial examined effects of metformin in overweight/obese patients with stage 0 -III breast cancer. The calculated ln (Ki67) showed non-significant changes when comparing metformin to placebo [67]. A different study with 200 participants randomized to metformin or placebo did not document any major difference in Ki67 and TUNEL levels (used for assessing cellular apoptosis) between the two groups. The study did note that TUNEL levels were higher in women without insulin resistance (metformin: $+4 \%$, interquartile range, IQR: 2-14, placebo: $+2 \%$, IQR: 0-7) as compared to those who had insulin resistance (metformin $+2 \%$, IQR: $0-6$, placebo +5\%, IQR: 0-15) [68].

Metformin is presently being evaluated as an anti-cancer agent for either monotherapy or combination therapy in endometrial cancer as well. The drugs that are currently being assessed in combination with metformin for the treatment of endometrial cancer are carboplatin, everolimus, letrozole, paclitaxel, and megestrol acetate. In conjunction with the clinical response, the effect of metformin on endometrial cancer is being studied through a wide variety of markers including Ki67, pS6, Akt, pAMPK, ERK1/2, histone H3, telomerase, topoisomerase II $\alpha$ and p27. The effect of using metformin on expression of estrogen receptor (ER) and progesterone receptor (PR) in cancer tissue of endometrial origin is also being investigated [69-71].

Metformin is being assessed in combination with various anticancer agents for the treatment of pancreatic cancer. The results from a phase II non-randomized trial showed that the combination of metformin with paclitaxel was not well tolerated, because, $42.1 \%$ patients experiencing grade 3-4 toxicities. A total of 31.6\% cases had to undergo metformin dose reduction after development of diarrhoea, on the other hand, one case experienced febrile neutropenia which was attributed to paclitaxel. This trial reported a median overall survival (OS) of 133 days and median progression free survival (PFS) as 43 days, but could not meet the disease control rate endpoint [72]. Another trial, consisting of 120 participants randomized to metformin or placebo group, noted that although the combination of metformin, gemcitabine and erlotinib was well tolerated, the 6 month survival rate was 55\% in metformin group and $66 \%$ in placebo group. Moreover, no significant difference was observed in PFS and median OS between metformin users and nonusers [73].

In case of treatment of prostate cancer, there are two trials that are using metformin as monotherapy and three in combination with different agents such as: abiraterone, docetaxel, and enzalutamide. Data from one trial, a single arm window of opportunity study, showed a significant reduction in Ki67 index and 4E BP-1 staining with no changes in pAMPK. Only three of 24 patients developed grade 3-4 toxicities, indicating that the treatment was overall well tolerated [74]. The effect of metformin therapy on PFS for prostate cancer is being assessed in two trials and in one other trial prostate specific antigen (PSA) response was evaluated. However, till date, no data is available on survival benefit.

There is one phase II trial of metformin use in non-small cell lung cancer (NSCLC) combined with stereotactic body radiotherapy. Very recently, a clinical study was carried out to study the clinical benefits of concurrent metformin and immune checkpoint inhibitors (ICIs) in NSCLC patients. This study was carried out as a retrospective review of 50 NSCLC patients receiving ICIs with metformin (cohort A) or without metformin (cohort B). Overall response rate and disease control rate were higher in cohort $\mathrm{A}$ ( 41.1 vs $30.7 \%, p=0.4$ and 70.5 vs $61.6 \%, p=0.5$, respectively). Median overall survival and progression-free survival were also higher in cohort A (11.5 vs 7.6 months, $p=0.5$ and 4.0 vs 3.0 months, $p=0.6$, respectively). On subset analysis (second-/thirdline ICIs), overall response rate, disease control rate, median overall survival, progression-free survival was also higher in cohort A. So, despite the small-sample size, this study have reported improved clinical outcomes in patients who received ICIs in combination with metformin [75]. Another clinical trial was carried out to assess the progression-free survival (PFS) in patients with advanced lung adenocarcinoma who received treatment with epidermal growth factor receptor- tyrosine kinase inhibitors (EGFR-TKIs) plus metformin compared with those who received EGFR-TKIs alone. Patients were randomly allocated to receive EGFR-TKIs (erlotinib hydrochloride, afatinib dimaleate, or gefitinib at standard dosage) plus metformin hydrochloride (500mg twice a day) or EGFR-TKIs alone. Treatment was continued until occurrence of intolerable toxic effects or withdrawal of consent. Between 21 months a total of 139 patients (mean [SD] age, 59.4 [12.0] years; 65.5\% female) were randomly assigned to receive EGFR-TKIs $(n=70)$ or EGFRTKIs plus metformin $(n=69)$. The median PFS was significantly longer in the EGFR-TKIs plus metformin group (13.1; 95\% CI, 9.816.3 months) compared with the EGFR-TKIs group (9.9; 95\% CI, 7.5-12.2 months) (hazard ratio, 0.60; 95\% CI, 0.40-0.94; $\mathrm{P}=.03$ ). The median OS was also significantly longer for patients receiving the combination therapy $(31.7$; $95 \% \mathrm{CI}, 20.5-42.8$ vs 17.5 ; $95 \% \mathrm{CI}$, 11.4-23.7 months; $\mathrm{P}=.02$ ) [76]. 
So, from these above clinical trial data, it is evident that metformin can be used in cancer treatment either as monotherapeutic agent or as a combination therapeutic agent with other chemotherapeutic drugs to improve overall survival rate (OS) and progression-free survival (PFS) of cancer patients.

\section{Discussion and Future Direction}

Epidemiological data has suggested the use of metformin with a decrease in the risk of developing cancer and a reduced cancer related mortality. The information that has been gathered from preclinical studies has provided encouraging evidence for anticancer mechanisms of metformin. It has also been suggested that metformin may well be used as a radiation sensitizer or an immunotherapy drug, in addition to a direct anti proliferative agent for the treatment of cancer. Now a days, researchers have concentrated on finding new target genes which may be used as a potential therapeutic target in cancer treatment. In our laboratory, we investigated that monoamine oxidase A (MAO-A), a gene which contributes to the resolution of inflammation [77] is also involved in the progression and metastasis of many different cancer cells [78]. Different previous researches have demonstrated that MAOA-mediated production of reactive oxygen species (ROS) can lead to DNA damage and oxidative injury of cells and may cause tumor initiation and progression. This shows that MAO-A might have a significant role in cancer. It has been reported that tumors from patients with aggressive prostate cancer (PCa) showed increased expression of MAO-A [79]. MAO-A also suppresses hepatocellular carcinoma (HCC) metastasis by inhibiting the adrenergic system and its transactivation of EGFR signalling and increasing MAO-A expression or enzyme activity may be a new approach that can be used for HCC therapy [80]. It has also been examined in prostate cancer cell line, that cytotoxic chemotherapy elevated the expression of MAO-A which in turn enhances cancer cell survival following docetaxel (chemotherapeutic drug) exposure [79]. MAO-A expression may be useful as a prognostic marker for cancer progression and efforts to modulate MAO-A expression may prove useful in the treatment of cancer [81]. Growing evidences thus suggest that MAO-A has an important role in epithelial to mesenchymal transition (EMT), promoting metastasis and aggressiveness in different cancer cell.

Recent findings from our group further suggest that IL-13/ IL-13R $\alpha 1 /$ Stat6 signalling pathway is involved in regulating the expression/activity of MAO-A in A549 lung epithelial carcinoma cells via a 15-lipoxygenase (15-LO)-dependent process involving peroxysome proliferator - activated receptor gamma (PPAR $\gamma$ ) and that may be the cause of promoting EMT in this specific lung cancer cells [80]. Similar result was obtained in HCT116 colorectal cancer cells and H1229 lung cancer cells where MAO-A is constitutively present, showed enhanced migration and invasion. On the other hand, MAO-A expression is induced by IL-13 in A549 lung epithelial carcinoma cells and expression of this induced MAO-A mediates ROS generation which is believed to have significant role on A549 cell migration, invasion and proliferation [82].

This finding from our group further strengthen our view that MAO-A could be an important target in lung cancer treatment.
Next, we asked the question whether metformin- the commercially available biguanide for treatment of diabetes and well known AMPK activator has any role in MAO-A regulation and finally in the regulation of cancer cell aggressiveness in lung cancer cells. An unpublished observation from our group suggests that metformin can inhibit MAO-A expression in IL-13-induced A549 lung carcinoma cells in a dose dependent manner. We found similar results in colorectal cancer cell line HCT116 after administration of metformin where MAO-A was constitutively expressed (our unpublished data). Moreover, both metformin and phenformin was equally holds responsible for reducing invasion and migration property in lung cancer cells (all these observations are now summarized in Figure 1). Additionally, we used a mixture of polyphenolic compounds which contains some known AMPK activators to check their effect on the expression of MAO-A in A549 cells. We further demonstrated that the mixture of polyphenolic compounds were able to inhibit MAO-A expression in dose dependent manner maintaining similar pattern as metformin (unpublished observations from our group). So, these observations likely suggest that metformin, the antidiabetic drug could be used in lung cancer treatment and MAO-A could be a potential therapeutic target for this purpose.

Considering our observation and other preclinical data, it is evident that MAO-A could serve as a very tempting molecular target of cancer treatment. A very recent clinical trial data of MAO-A inhibitor Phenelzine in patients with biochemical recurrent castrate-sensitive prostate cancer has suggested that therapies directed at MAO-A may represent a new avenue for treatment in patients with recurrent prostate cancer [83]. Apart from using only MAO-A inhibitors or metformin for treatment of cancer in different clinical trials, both can be used in combination therapy for better result and efficacy. Moreover, considering the potential of polyphenolic mixture to regulate MAO-A expression, polyphenolic mixture in combination with metformin could serve as a very promising therapy for the treatment of different forms of cancer in future.

\section{Conclusion}

A strong base of epidemiological, laboratory and pre-clinical data has prompted attempts to probe the anti-cancer effects of metformin through clinical trials. Metformin has been shown to have a commendable effect on different markers of tumor proliferation, invasion and metastasis. But it is still unclear that whether it has any significant effect on overall survival rates. It is more important to find better histology and the appropriate stage of tumors for utilizing metformin therapy. Marking different new molecular targets involved in this process could also be an effective measure for further improvement in cancer treatment. The potential use of metformin as an immunotherapy agent needs to be substantiated with further evidence to ascertain possible benefits in future.

\section{Acknowledgement}

The authors would like to acknowledge all the lab members for their support during this work and finally acknowledge the Department of Biotechnology, NIT Durgapur for extending facilities for research work. The unpublished research work was supported 
by the department of higher education, Science and Technology and Biotechnology, Govt. of West Bengal and Department of Biotechnology, Govt of India to AB. CD was supported by DBT-RA fellowship and PD was supported by UGC fellowship.

\section{Conflict of Interest}

The authors declare that they have no conflict of interest with the contents of this article.

\section{References}

1. World Health Organization/ Home/ Health topics/ Cancer, Home/ Newsroom /Fact sheets/ Detail/ Cancer, Global Cancer Observatory (GCO).

2. Kasznicki J, Sliwinska A, Drzewoski J (2014) Metformin in cancer prevention and therapy. Ann Transl Med 2(6): 57.

3. Dowling RJ, Goodwin PJ, Stambolic V (2011) Understanding the benefit of metformin use in cancer treatment. BMC Med 9: 33.

4. Malek M, Aghili R, Emami Z, Khamseh ME (2013) Risk of cancer in diabetes: the effect of metformin. ISRN Endocrinol 2013: 636927.

5. Gul W (2016) Metformin: Methods of analysis and its role in lowering the risk of cancer. J Bioequiv Availab 8: 254-259.

6. Dawes G, Mott J (1950) Circulatory and respiratory reflexes caused by aromatic guanidines. Br J Pharmacol Chemother 5(1): 65-76.

7. Zaharenko L (2015) Pharmacogenetics of efficiency and tolerance of the peroral antidiabetic drug metformin. Riga: University of Latvia, Riga, Latvia.

8. Kourelis TV, Siegel RD (2012) Metformin and cancer: New applications for an old drug. Med Oncol 29(2): 1314-1327.

9. Spillane SC, Bennett K, Sharp L, Barron TI (2013) A cohort study of metformin exposure and survival in patients with stage I-III colorectal cancer. Cancer Epidemiol Prev Biomarkers 22(8): 1364-1373.

10. Foretz M, Guigas B, Bertrand L, Pollak M, Viollet B (2014) Metformin From mechanisms of action to therapies. Cell Metab 20(6): 953-966.

11. Muaddi H, Chowdhury S, Vellanki R, Zamiara P, Koritzinsky M (2013) Contributions of AMPK and p53 dependent signaling to radiation response in the presence of metformin. Radiother Oncol 108(3): 446450.

12. Cantoria MJ, Patel H, Boros LG, Meuillet EJ (2014) Metformin and pancreatic cancer metabolism. pancreatic cancer-insights into molecular mechanisms and novel approaches to early detection and treatment. London, UK.

13. Del Barco S, Vazquez-Martin A, Cufí S, Oliveras-Ferraros C, BoschBarrera J, et al. (2011) Metformin: multi-faceted protection against cancer. Oncotarget 2(12): 896-917.

14. Graham GG, Punt J, Arora M, Day RO, Doogue MP, et al. (2011) Clinical pharmacokinetics of metformin. Clin Pharmacokinet 50(2) : 81-88.

15. El-Mir MY, Nogueira V, Fontaine E, Avéret N, Rigoulet M, et al. (2000) Dimethylbiguanide inhibits cell respiration via an indirect effect targeted on the respiratory chain complex I. J Biol Chem 275(1): 223-228.

16. Stephenne X, Foretz M, Taleux N, van der Zon GC, Sokal E, et al. (2011) Metformin activates AMP-activated protein kinase in primary human hepatocytes by decreasing cellular energy status. Diabetologia 54(12): 3101-3110.

17. Samuel SM, Varghese E, Varghese S, Busselberg D (2018) Challenges and perspectives in the treatment of diabetes associated breast cancer. Cancer Treat Rev 70: 98-111.

18. Christensen MM, Hojlund K, Hother-Nielsen O, Stage TB, Damkier P, et al. (2015) Steady-state pharmacokinetics of metformin is independent of the OCT1 genotype in healthy volunteers. Eur J Clin Pharm 71(6): 691697.

19. Kinaan M, Ding H, Triggle CR (2015) Metformin: An old drug for the treatment of diabetes but a new drug for the protection of the endothelium. Med Princ Pract 24(5): 401-415.

20. Correia S, Carvalho C, Santos MS, Seica R, Oliveira CR, et al. (2008) Mechanisms of action of metformin in type 2 diabetes and associated complications: An overview. Mini Rev Med Chem 8(13): 1343-1354.

21. Liang X, Giacomini KM (2017) Transporters involved in metformin pharmacokinetics and treatment response. J Pharm Sci 106(9): 22452250.

22. Choi MK, Jin QR, Jin HE, Shim CK, Cho DY, et al. (2007) Effects of tetra alkyl ammonium compounds with different affinities for organic cation transporters on the pharmacokinetics of metformin. Biopharm Drug Dispos 28(9): 501-510.

23. Kimura N, Masuda S, Tanihara Y, Ueo H, Okuda M, et al. (2005) Metformin is a superior substrate for renal organic cation transporter OCT2 rather than hepatic OCT1. Drug Metab Pharmacokinet 20(5): 379-386.

24. Li S, Chen Y, Zhang S, More SS, Huang X, et al. (2011) Role of organic cation transporter 1, OCT1 in the pharmacokinetics and toxicity of cisdiammine (pyridine) chloroplatinum(II) and oxaliplatin in mice. Pharm Res 28(3): 610-625.

25. Chen EC, Liang X, Yee SW, Geier EG, Stocker SL, et al. (2015) Targeted disruption of organic cation transporter 3 attenuates the pharmacologic response to metformin. Mol Pharmacol 88(1): 75-83.

26. Chen Y, Teranishi K, Li S, Yee SW, Hesselson S, et al. (2009) Genetic variants in multidrug and toxic compound extrusion-1, hMATE1, alter transport function. Pharm J 9(2): 127-136.

27. Masuda S, Terada T, Yonezawa A, Tanihara Y, Kishimoto K, et al. (2006) Identification and functional characterization of a new human kidneyspecific $\mathrm{H}+$ /organic cation antiporter, kidney-specific multidrug and toxin extrusion 2. J Am Soc Nephrol 17(8): 2127-2135.

28. Zhou M, Xia L, Wang J (2007) Metformin transport by a newly cloned proton-stimulated organic cation transporter (plasma membrane monoamine transporter) expressed in human intestine. Drug Metab Dispos 35(10): 1956-1962.

29. Liang X, Chien HC, Yee SW, Giacomini MM, Chen EC, etal. (2015) Metformin is a substrate and inhibitor of the human thiamine transporter, THTR2(SLC19A3). Mol Pharm 12(12): 4301-4310.

30. Shu Y, Brown C, Castro RA, Shi RJ, Lin ET, et al. (2008) Effect of genetic variation in the organic cation transporter 1, OCT1, on metformin pharmacokinetics. Clin Pharm 83(2): 273-280.

31. Shu Y, Sheardown SA, Brown C, Owen RP, Zhang S, et al. (2007) Effect of genetic variation in the organic cation transporter 1 (OCT1) on metformin action. J Clin Investig 117(5): 1422-1431.

32. Rahmani J, Manzari N, Thompson J, Gudi SK, Chhabra M, et al. (2019) The effect of metformin on biomarkers associated with breast cancer outcomes: A systematic review, meta-analysis, and dose-response of randomized clinical trials. Clin Transl Oncol 22(1): 37-49.

33. Sosnicki S, Kapral M, Weglarz L (2016) Molecular targets of metformin antitumor action. Pharmacol Rep 68(5): 918-925.

34. Daugan M, Dufaÿ Wojcicki A, d'Hayer B, Boudy V (2016) Metformin: An anti-diabetic drug to fight cancer. Pharmacol Res 113(Pt A): 675-685.

35. Le A, Lane AN, Hamaker M, Bose S, Gouw A, et al. (2012) Glucoseindependent glutamine metabolism via TCA cycling for proliferation and survival in B cells. Cell Metab 15(1): 110-121.

36. Metallo CM, Gameiro PA, Bell EL, Mattaini KR, Yang J, et al. (2012) Reductive glutamine metabolism by IDH1 mediates lipogenesis under hypoxia. Nature 481(7381): 380-384 
37. Samuel SM, Ghosh S, Majeed Y, Arunachalam G, Emara MM, et al. (2017) Metformin represses glucose starvation induced autophagic response in microvascular endothelial cells and promotes cell death. Biochem Pharmacol 132: 118-132.

38. Hawley SA, Gadalla AE, Olsen GS, Hardie DG (2002) The antidiabetic drug metformin activates the amp-activated protein kinase cascade via an adenine nucleotide-independent mechanism. Diabetes 51(8): 2420 2425 .

39. Zhou G, Myers R, Li Y, Chen Y, Shen X, et al. (2001) Role of AMP-activated protein kinase in mechanism of metformin action. J Clin Investig 108(8): 1167-1174

40. Meng S, Cao J, He Q Xiong L, Chang E, et al. (2015) Metformin activates AMP-activated protein kinase by promoting formation of the heterotrimeric complex. J Biol Chem 290(6): 3793-3802.

41. Howell JJ, Hellberg K, Turner M, Talbott G, Kolar MJ, et al. (2017) Metformin inhibits hepatic mTORC1 signaling via dose-dependent mechanisms involving ampk and the tsc complex. Cell Metab 25(2): 463471.

42. Sabnis HS, Somasagara RR, Bunting KD (2017) Targeting MYC dependence by metabolic inhibitors in cancer. Genes 8(4): 114.

43. Shen P, Reineke LC, Knutsen E, Chen M, Pichler M, et al. (2018) Metformin blocks MYC protein synthesis in colorectal cancer via mTOR-4EBP-eIF4E and MNK1-eIF4G-eIF4E signaling. Mol Oncol 12(11): 1856-1870.

44. Sekino N, Kano M, Matsumoto Y, Sakata H, Akutsu Y, et al. (2018) Antitumor effects of metformin are a result of inhibiting nuclear factor kappa B nuclear translocation in esophageal squamous cell carcinoma Cancer Sci 109(4): 1066-1074.

45. Xu S, Yang Z, Jin P, Yang X, Li X, et al. (2018) Metformin suppresses tumor progression by inactivating stromal fibroblasts in ovarian cancer. Mol Cancer Ther 17(6): 1291-1302.

46. Li P, Zhao M, Parris A, Feng X, Yang X (2015) p53 is required for metformin-induced growth inhibition, senescence and apoptosis in breast cancer cells. Biochem Biophys Res Commun 464(4): 1267-1274.

47. Chen L, Ahmad N, Liu X(2016) Combining p53 stabilizers with metformin induces synergistic apoptosis through regulation of energy metabolism in castration-resistant prostate cancer. Cell Cycle 15(6): 840-849.

48. Yi Y, Zhang W, Yi J, Xiao ZX (2019) Role of p53 Family Proteins in Metformin Anti-Cancer Activities. J Cancer 10(11): 2434-2442.

49. Yi G, He Z, Zhou X, Xian L, Yuan T, et al. (2013) Low concentration of metformin induces a p53-dependent senescence in hepatoma cells via activation of the AMPK pathway. Int J Oncol 43(5): 1503-1510.

50. Noren Hooten N, Martin-Montalvo A, Dluzen DF, Zhang Y, Bernier M, et al. (2016) Metformin-mediated increase in DICER1 regulates microRNA expression and cellular senescence. Aging Cell 15(3): 572-581.

51. Fullerton MD, Galic S, Marcinko K, Sikkema S, Pulinilkunnil T, et al (2013) Single phosphorylation sites in Acc1 and Acc2 regulate lipid homeostasis and the insulin-sensitizing effects of metformin. Nat Med 19(12): 1649.

52. Gupta S, Roy A, Dwarakanath BS (2017) Metabolic cooperation and competition in the tumor Microenvironment: Implications for Therapy. Front Oncol 7: 68 .

53. Wolff NC, Vega-Rubin-de-Celis S, Xie XJ, Castrillon DH, Kabbani W, et al. (2011) Cell-type-dependent regulation of mTORC1 by REDD1 and the tumor suppressors TSC1/TSC2 and LKB1 in response to hypoxia. Mol Cell Biol 31(9): 1870-1884.

54. Katiyar S, Liu E, Knutzen CA, Lang ES, Lombardo CR, et al. (2009) REDD1, an inhibitor of mTOR signalling, is regulated by the CUL4A-DDB1 ubiquitin ligase. EMBO Rep 10(8): 866-872.

55. Tirado-Hurtado I, Fajardo W, Pinto JA (2018) DNA damage inducible transcript 4 gene: The switch of the metabolism as potential target in cancer. Front Oncol 8: 106.
56. De Young MP, Horak P, Sofer A, Sgroi D, Ellisen LW (2008) Hypoxia regulates TSC1/2 mTOR signaling and tumor suppression through REDD1-mediated 14-3-3 shuttling. Genes Dev 22(2): 239-251.

57. Ben Sahra I, Regazzetti C, Robert G, Laurent K, Le Marchand-Brustel Y, et al. (2011) Metformin, independent of AMPK, induces mTOR inhibition and cell-cycle arrest through REDD1. Cancer Res 71(13): 4366-4372.

58. Lei Y, Yi Y, Liu Y, Liu X, Keller ET, et al. (2017) Metformin targets multiple signalling pathways in cancer. Chin J Cancer 36(1): 17.

59. Kalender A, Selvaraj A, Kim SY, Gulati P, Brûlé S, et al. (2010) Metformin, independent of AMPK, inhibits mTORC1 in a rag GTPase-dependent manner. Cell Metab 11(5): 390-401.

60. Fu K, Jiang C, Huang X, Chan WC, McKeithan T (2013) The combination of 2-DG and metformin inhibits the mTORC1 pathway and suppresses aggressive B cell lymphoma growth and survival. Blood 122(21): 1665.

61. Avalle L, Camporeale A, Camperi A, Poli V (2017) STAT3 in cancer: A double edged sword. Cytokine 98: 42-50.

62. Huynh J, Chand A, Gough D, Ernst M (2019) Therapeutically exploiting STAT3 activity in cancer-using tissue repair as a road map. Nat Rev Cancer 19(2): 82-96.

63. Niraula S, Dowling RJ, Ennis M, Chang MC, Done SJ, et al. (2012) Metformin in early breast cancer: A prospective window of opportunity neoadjuvant study. Breast Cancer Res Treat 135(3): 821-830.

64. Wallace TM, Matthews DR (2002) The assessment of insulin resistance in man. Diabet Med 19(7): 527-534.

65. Hadad SM, Coates P, Jordan LB, Dowling RJ, Chang MC, et al. (2015) Evidence for biological effects of metformin in operable breast cancer: biomarker analysis in a pre-operative window of opportunity randomized trial. Breast Cancer Res Treat 150(1): 149-155.

66. Bonanni B, Puntoni M, Cazzaniga M, Pruneri G, Serrano D, et al. (2012) Dual effect of metformin on breast cancer proliferation in a randomized pre-surgical trial. J Clin Oncol 30(21): 2593-2600.

67. Kalinsky K, Crew KD, Refice S, Xiao T, Wang A, et al. (2014) Presurgical trial of metformin in overweight and obese patients with newly diagnosed breast cancer. Cancer Invest 32(4): 150-157.

68. Cazzaniga M, DeCensi A, Pruneri G, Puntoni M, Bottiglieri L, et al. (2013) The effect of metformin on apoptosis in a breast cancer presurgical trial. Br J Cancer 109(11): 2792-2797.

69. Schuler KM, Rambally BS, DiFurio MJ, Sampey BP, Gehrig PA, et al. (2015) Antiproliferative and metabolic effects of metformin in a preoperative window clinical trial for endometrial cancer. Cancer Med 4(2): 161-173.

70. Laskov I, Drudi L, Beauchamp MC, Yasmeen A, Ferenczy A, et al. (2014) Anti-diabetic doses of metformin decrease proliferation markers in tumors of patients with endometrial cancer. Gynecol Oncol 134(3): 607614.

71. Mitsuhashi A, Kiyokawa T, Sato Y, Shozu M (2014) Effects of metformin on endometrial cancer cell growth in vivo: A preoperative prospective trial. Cancer 120(19): 2986-2995.

72. De Celis Ferrari ACR, Pfiffer TEF, Alex AK, Nebuloni DR, Carneiro AQ et al. (2015) Phase II trial of metformin and paclitaxel for patients with gemcitabine refractory advanced adenocarcinoma of the pancreas. Ecancermedicalscience 9: 536.

73. Wilmink J, Kordes S, Zwinderman K, Mathot R, Punt CJA, et al. (2014) A phase II randomized, placebo controlled study to evaluate the efficacy of the combination of gemcitabine, erlotinib, and metformin in patients with locally advanced or metastatic pancreatic cancer. ASCO Meeting Abstracts 32(15): 4021.

74. Joshua AM, Zannella VE, Downes MR, Bowes B, Hersey K, et al. (2014) A pilot 'window of opportunity' neoadjuvant study of metformin in localised prostate cancer. Prostate Cancer Prostatic Dis 17(3): 252-258. 
75. Afzal MZ, Dragnev K, Sarwar T, Shirai K (2019) Clinical outcomes in non-small-cell lung cancer patients receiving concurrent metformin and immune checkpoint inhibitors. Lung Cancer Manag 8(2): eISSN 17581974.

76. Arrieta O, Barrón F, Salinas Padilla MA, Alejandro A, Laura A, et al (2019) Effect of metformin plus tyrosine kinase inhibitors compared with tyrosine kinase inhibitors alone in patients with epidermal growth factor receptor-mutated lung adenocarcinoma: A phase 2 randomized clinical trial. JAMA Oncol 5(11): e192553.

77. Cathcart MK, Bhattacharjee A (2014) Monoamine oxidase A (MAO-A) A signature marker of alternatively activated monocytes/macrophages. Inflamm Cell Signal 1(4): 152-159.

78. Biswas P, Dhabal S, Das P, Swaroop S, Tuhina P, et al. (2018) Role of Monoamine oxidase A (MAO-A) in cancer progression and metastasis (Review). Cancer Cell \& Microenvironment 5: e1623.

79. Wu JB, Shao C, Li X, Li Q Hu P, et al. (2014) Monoamine oxidase A mediates prostate tumorigenesis and cancer metastasis. J Clin Invest 124(7): 2891-2908.
80. Li J, Yang XM, Wang YH, Feng MX, Liu XJ, et al. (2014) Monoamine oxidase A suppresses hepatocellular carcinoma metastasis by inhibiting the adrenergic system and its transactivation of EGFR signalling. J Hepatol 60(6): 1225-1234.

81. Gordon RR, Wu M, Huang CY, Harris WP, Sim HG, et al. (2014) Chemotherapy-induced monoamine oxidase expression in prostate carcinoma functions as a cytoprotective resistance enzyme and associates with clinical outcomes. PLoS One 9(9): e104271.

82. Dhabal S, Das P, Biswas P, Kumari P, Yakubenko VP, et al. (2018) Regulation of monoamine oxidase A (MAO-A) expression, activity and function in IL-13-stimulated monocytes and A549 lung carcinoma cells. J Biol Chem 293(36): 14040-14064.

83. Gross ME, Agus DB, Dorff TB, Pinski JK, Quinn DI, et al. (2020) Phase 2 trial of monoamine oxidase inhibitor phenelzine in biochemical recurrent prostate cancer. Prostate Cancer Prostatic Dis. 